\title{
Technè
}

La science au service de l'histoire de l'art et de la préservation des biens culturels

44 | 2016

Archives de l'humanité : les restes humains patrimonialisés

\section{L'utilisation des isotopes en archéologie et en anthropologie}

Use of isotopes for archaeology and anthropology

\section{Fabrice Demeter}

\section{(2) OpenEdition}

10 Journals

\section{Édition électronique}

URL : http://journals.openedition.org/techne/1090

DOI : 10.4000/techne.1090

ISSN : 2534-5168

\section{Éditeur}

C2RMF

\section{Édition imprimée}

Date de publication : 1 novembre 2016

Pagination : 71-73

ISBN : 978-2-7118-6339-6

ISSN : 1254-7867

Référence électronique

Fabrice Demeter, «L'utilisation des isotopes en archéologie et en anthropologie », Technè [En ligne], 44 | 2016, mis en ligne le 19 décembre 2019, consulté le 23 juillet 2020. URL : http://

journals.openedition.org/techne/1090; DOI : https://doi.org/10.4000/techne.1090

\section{(C) $(\Theta \Theta \Theta$}

La revue Technè. La science au service de l'histoire de l'art et de la préservation des biens culturels est mise à disposition selon les termes de la Licence Creative Commons Attribution - Pas d'Utilisation

Commerciale - Pas de Modification 4.0 International. 
Fabrice Demeter

\section{L'utilisation des isotopes en archéologie et en anthropologie}

Use of isotopes for archaeology

and anthropology

Résumé. Les isotopes sont présents partout dans l'environnement. Ils servent à dater les matériaux organiques lorsqu'ils sont radioactifs ou à renseigner sur l'alimentation et l'origine géographique des humains et des animaux lorsqu'ils sont stables.

Mots-clés. Isotopes, alimentation, migrations humaines.
Abstract. Isotopes are present everywhere in the environment. They are used to date organic materials when they are radioactive or to gather information about food and the geographical origin of humans and animals when they are stable.

Keywords. Isotopes, food, human migrations.

\section{Les isotopes, pour quoi faire?}

Les isotopes sont présents partout dans l'environnement et se retrouvent dans les plantes par l'intermédiaire des sédiments et de l'eau, puis dans les tissus animaux (et donc dans ceux de l'homme) à travers l'alimentation, la boisson et la respiration $^{1}$. L'étude des isotopes radioactifs, comme le célèbre carbone $14\left({ }^{14} \mathrm{C}\right)$ permet de dater les matériaux qui en contiennent, comme le bois, les fibres végétales (tissus) et l'os. L'analyse des isotopes stables, comme ceux du carbone et de l'azote, permet d'étudier les habitudes alimentaires d'un individu ou d'un groupe d'individus ${ }^{2}$ et de déterminer l'endroit dans lequel une personne a grandi ou vécu les 20 à 25 dernières années de sa vie ${ }^{3}$. Tous ces isotopes sont donc des éléments extrêmement utiles pour les sciences archéologiques et anthropologiques ${ }^{4}$.

\section{Quels sont les isotopes les plus étudiés?}

Les éléments chimiques les plus fréquemment employés pour les analyses isotopiques sont le carbone, l'azote, l'hydrogène, l'oxygène et le strontium.

Le carbone ( $\mathbf{C}$ ) est présent dans l'air, l'eau et la terre, et est absorbé par les plantes lors de la photosynthèse, soit la transformation de la lumière du soleil en énergie utilisable ${ }^{5}$. Les différentes espèces de plantes absorbent le carbone en fonction de leurs caractéristiques spécifiques et du climat dans lequel elles vivent. Par exemple, les cellules des plantes tropicales ont des ratios isotopiques différents de celles des plantes des climats tempérés. Les animaux et êtres humains consomment des plantes. Par conséquent, le carbone peut être utilisé pour déterminer le type d'environnement dans lequel un animal ou une personne a vécu, ainsi que son régime alimentaire ${ }^{6}$.

L'azote (N) est aussi présent dans l'atmosphère, mais il est absorbé par les plantes par fixation. Le ratio isotopique d'azote varie entre les plantes et les animaux qui les consomment, puis entre ces derniers et les autres animaux qui les mangent. Par conséquent, les ratios isotopiques d'azote peuvent être utilisés pour déterminer la place d'un organisme dans la chaîne alimentaire (niveau trophique) : herbivore, carnivore ou omnivore ${ }^{7}$. De cette manière, un herbivore aura un collagène osseux dont la composition isotopique est enrichie par rapport à la part protéique de son alimentation. Il en est de même pour un carnivore par rapport à sa proie. L'analyse des ${ }^{13} \mathrm{C}$ et ${ }^{15} \mathrm{~N}$ du collagène osseux des populations va apporter ainsi des informations sur l'environnement dans lequel elles auront puisé leurs ressources, ainsi que sur le niveau trophique auquel il appartient ${ }^{8}$.

L'hydrogène $(\mathbf{H})$ et l'oxygène $(\mathbf{O})$ sont les composants de l'eau, ils sont donc présents dans l'environnement sous diverses formes ${ }^{9}$. Pour ces deux éléments, les ratios isotopiques varient en fonction de la température et du climat. Les êtres humains consomment de l'eau. Par comparaison, il est possible de déterminer si un individu a vécu dans différentes régions géographiques au cours de sa vie, ou si plusieurs individus proviennent du même endroit (et ont consommé la même eau). Bien que l'hydrogène et l'oxygène puissent être très utiles pour les études isotopiques, les techniques d'analyse sont toujours en cours de développement et les résultats doivent être interprétés prudemment.

Le strontium (Sr) est un métal qui regroupe plusieurs isotopes stables et radioactifs. Il est abondant dans la nature et se trouve surtout dans les roches et les sédiments volcaniques. Avec l'érosion des sédiments et leur dispersion dans 
l'eau et les ressources alimentaires, il est absorbé par l'organisme qui l'incorpore dans les tissus osseux, de la même manière que le calcium. Le ratio isotopique du strontium varie d'une région géographique à l'autre. Par conséquent, l'analyse des ratios isotopiques du strontium des os peut permettre de déterminer l'origine géographique d'une personne ou de distinguer des squelettes retrouvés ensemble mais ayant appartenu à des individus d'origines géographiques différentes.

\section{Quels types de tissus utiliser ?}

Les os et les dents sont les tissus les plus fréquemment analysés, car ils sont durs et se conservent longtemps dans les contextes archéologique et médico-légal. L'os est formé de deux composants : une matrice organique majoritairement composée de collagène, et une matrice minérale inorganique composée surtout de phosphates de calcium. Le collagène est une protéine fibreuse, la plus abondante chez les mammifères ${ }^{10}$. Comme pour le cheveu, la protéine de collagène de l'os est constituée de différents atomes, notamment de carbone et d'azote, dont la composition isotopique $\left({ }^{13} \mathrm{C}\right.$ et $\left.{ }^{15} \mathrm{~N}\right)$ est liée à celle de la part protéique de l'alimentation ${ }^{11}$. Dans la nature, les végétaux possèdent des ${ }^{13} \mathrm{C}$ et ${ }^{15} \mathrm{~N}$ spécifiques à leur environnement (marin, terrestre, aride, tempéré...), leur photosynthèse (C3, C4 ou CAM) ${ }^{12}$ et leur espèce (e.g légumineuse ou non légumineuse). L'os est un tissu vivant qui se renouvelle en permanence lorsque nous grandissons et vieillissons. Cependant, ce processus est très lent, et l'os cortical dense reflète approximativement les 10 à 15 dernières années de vie d'un individu.

Les dents sont aussi composées de matériaux organiques et minéraux, mais contrairement à l'os, l'émail dentaire ne se renouvelle pas. Par conséquent, les dents sont très utiles lorsque l'on veut déterminer l'environnement des premières années de vie d'un individu (au moment où ses dents se sont formées). De plus, la comparaison des dents et des os d'un même individu permet de déterminer si une personne a migré d'une région à l'autre depuis son enfance : les dents montrent le lieu de vie pendant l'enfance, et les os le lieu de vie pendant les années qui ont précédé le décès.

Les cheveux qui sont composés de deux protéines, la kératine et le collagène, ainsi que les ongles peuvent aussi être utiles aux analyses isotopiques. Bien qu'ils soient plus fragiles que les os, ils se forment à un rythme connu et reflètent le passé récent des individus. Ils peuvent donc permettre de déterminer si un individu s'est déplacé d'une région à l'autre récemment, ou des changements alimentaires saisonniers ${ }^{13}$.

Finalement, le sang et les tissus mous peuvent aussi être employés pour les analyses isotopiques. Cependant, ils se décomposent rapidement et sont fortement sujets à la

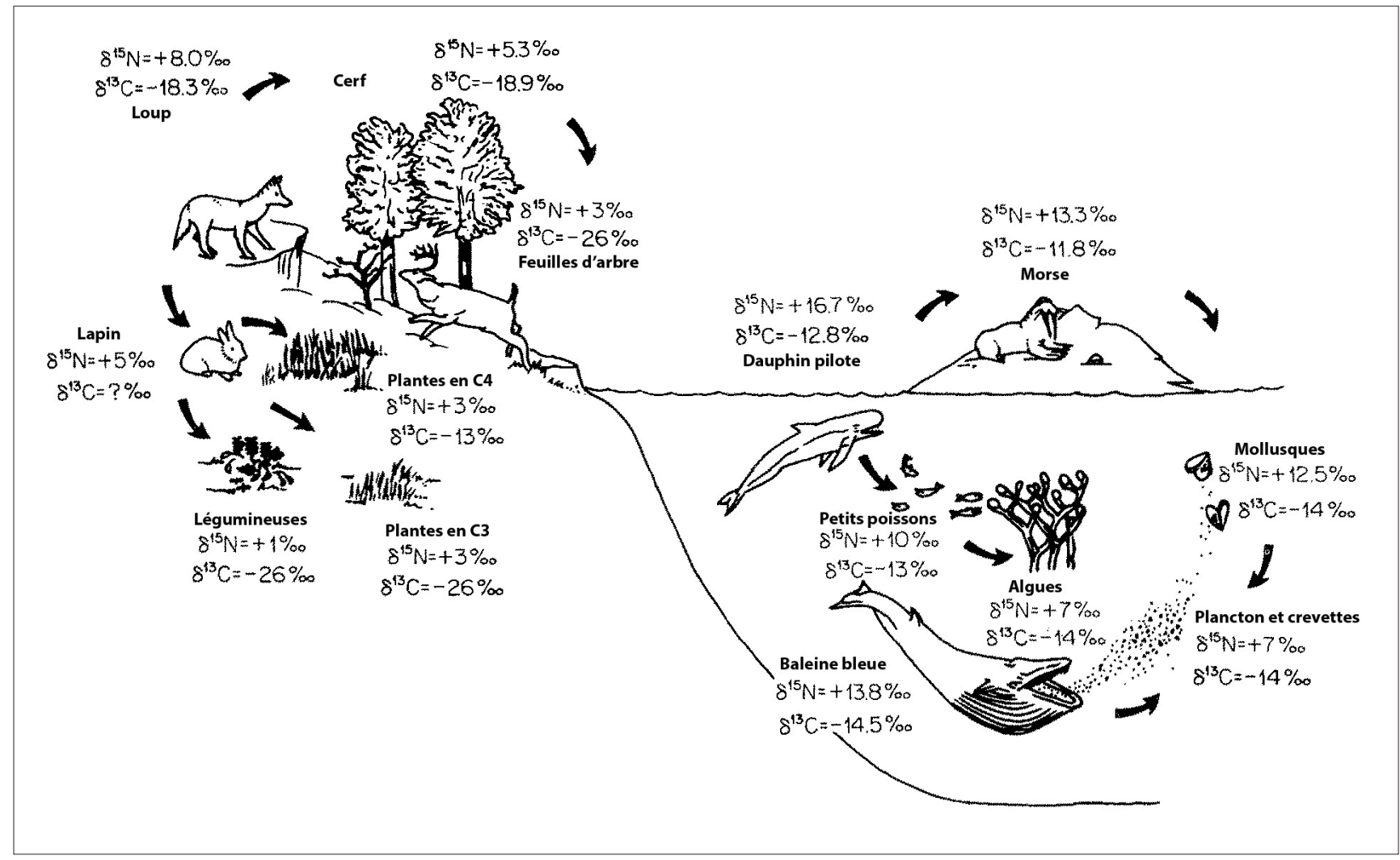

Fig. 1. Distribution simplifiée des ratios d'isotopes stables du carbone et de l'azote dans la biosphère (adapté d'après Shoeninger et Moore, 1992). 
contamination. L'abondance de l'isotope lourd du carbone et de l'azote, respectivement ${ }^{13} \mathrm{C}$ et ${ }^{15} \mathrm{~N}$, reflète l'alimentation des individus : l'analyse isotopique peut donc montrer si la personne est végétarienne ou si elle consomme beaucoup de viande. De la même manière, l'abondance des isotopes lourds d'hydrogène et d'oxygène dans les tissus, respectivement $2 \mathrm{H}$ et 180 , reflète l'eau consommée par les individus (par le biais de boissons et d'aliments comme les fruits et les légumes).

Notes

1. Bowen, 2010.

2. Ambrose, Norr, 1993 ; Clementz et al., 2009.

3. Balasse et al., 2002.

4. Shoeninger, Moore, 1992 ; Knipper et al., 2015.

5. Drucker, D., Bocherens, H., 2004.

6. Dufour et al., 2014.

7. Deniro, Epstein, 1978 ; Krueger, Sullivan, 1984.

8. Bocherens et al., 1999.

9. Bowen et al., 2005.

10. Campbell, 1995.

11. Ambrose, Norr, 1993.

12. Le mécanisme de photosynthèse

en C3 3 correspond au mécanisme "de base " de $95 \%$ des plantes vertes. Dans les prairies des régions subtropicales à tropicales avec une forte insolation et une saison des pluies chaudes, certaines plantes ont développé des alternatives aux limitations imposées par le milieu, afin de préserver une activité photosynthétique (herbacées) appelée photosynthèse en C4. En milieu aride, une autre photosynthèse s'est développée, la photosynthèse CAM (plantes grasses, ananas).

13. Macko et al., 1999 ; O’Connel, Hedges, 1999.

\section{Bibliographie}

Ambrose S. H., Norr L., 1993, "Experimental evidence for the relationship of the carbon isotope ratios of whole diet and dietary protein to those of bone collagen and carbonate", in Prehistoric human bone Archaeology at the molecular level, (ed) J. B. Lambert, G. Grupe (Berlin: Springer-Verlag), p. 1-37.

Balasse M., Ambrose S. H., Smith A. B., Price T. D., 2002, "The seasonal mobility model for prehistoric herders in the south-western Cape of South Africa assessed by isotopic analysis of sheep tooth enamel", Journal of Archaeological Science 29: 917-932.

Bocherens H., Mariotti A., Lévêque F., Vandermeersch B., Guadelli J.-L., 1999 «Conservation des signatures isotopiques du collagène d'os et de dents du pléistocène supérieur (SaintCésaire, France) : implications pour les reconstitutions des régimes alimentaires des néandertaliens ", Bull. Mém. Soc. Anthrop. Paris, Nouvelle Série, tome 11 fascicule 3-4, 289-305.

Bowen G. J., 2010, "Isoscapes: Spatial pattern in isotopic biogeochemistry", Annual Review of Earth and Planetery Sciences 38, 161-187.

Bowen G. J., Winter D. A., Spero H. J., Zierenberg R. A., Reeder M. D., Cerling T. E. and Ehleringer J. R., 2005, "Stable hydrogen and oxygen isotope ratios of bottled waters of the world", Rapid Communications in Mass Spectrometry 19, 3442-3450.

Campbell N. A., 1995, Biologie (Bruxelles: de Boeck), p. 1190

Clementz M. T., Fox-Dobbs K., Wheatley P. V., Koch P. L., Doak D. F., 2009, "Revisiting old bones: coupled carbon isotope analysis of bioapatite and collagen as an ecological and palaeoecological tool", Geological Journal 44: 605-620.

Deniro M. J., Epstein, S., 1978, "Influence of diet on the distribution of carbon isotopes in animals", Geochimica et Cosmochimica Acta, 42, 495-506.

Drucker D., Bocherens H., 2004, "Carbon and nitrogen stable isotopes as tracers of change in diet breadth during Middle and Upper Palaeolithic in Europe", International Journal of Osteoarchaeology, 14, 162-177.

Dufour E., Goepfert N., Gutiérrez Léon B. Chauchat C., Franco Jordán R. et al., 2014, "Pastoralism in Northern Peru during Pre-Hispanic Times: Insights from the Mochica Period (100-800 AD) Based on Stable Isotopic Analysis of Domestic Camelids", PLoS ONE 9(1): e87559.

Knipper C. et al., 2015, "Superior in Life Superior in Death. Dietary Distinction of Central European Prehistoric and Medieval Elites", Current Anthropology 56: 579-589.

Krueger H. W., Sullivan, 1984, "Models for carbon isotope fractionation between diet and bone", in Stable isotopes in nutrition, (ed) J. Turnlund et P. Johnson (Washington: American Chemical Society Symposium Series 258), p. 205-220.

Macko S. A., Engel M. H., Andrusevich V., Lubec G., O'Connell T. C., Hedges R. E., 1999, "Documenting the diet in ancient human populations through stable isotope analysis of hair", Philos Trans R Soc Lond B Biol Sci. : 354(1379):65-75; discussion 75-6

O'Connel T. C., Hedges E. R., 1999, "Investigations into the effect of diet on modern human hair isotopic values", Am J Phys Anthropol. : 108(4):409-25.

Shoeninger M. J., Moore, K., 1992, "Bone stable isotope studies in archaeology", J. W. Prehist. : 6(2): 247-296. 